\title{
Correction to "Glutathione S-Transferase P Influences Redox Homeostasis and Response to Drugs that Induce the Unfolded Protein Response in Zebrafish"
}

The above article [Zhang L, Kim S-H, Park K-H, Townsend DM, Tew KD, Ye Z-W, and Jie Z (2021) J Pharmacol Exp Ther, 377: 121-132; DOI: https://doi.org/10.1124/jpet.120.000417], was published with several errors introduced by the compositor in the author byline. The correct byline for the article is Leilei Zhang, Seok-Hyung Kim, Ki-Hoon Park, Zhi-wei Ye, Jie Zhang, Danyelle M. Townsend, and Kenneth D. Tew.

The PDF and HTML versions of the article have been corrected.

The compositor apologizes for any inconvenience caused by these errors. 